\title{
NUT midline carcinoma mimicking a germ cell tumor: a case report
}

\author{
Yohei Harada ${ }^{12^{*}}$ (D), Takafumi Koyama ${ }^{1}$, Kengo Takeuchi ${ }^{3,4}$, Kazufusa Shoji $^{5}$, Kazuei Hoshi ${ }^{6}$ and Yu Oyama ${ }^{1}$
}

\begin{abstract}
Background: NUT midline carcinoma (NMC) is a rare and highly aggressive malignancy. Although more information on NMC has been recently accumulating in the literature, most oncologists and pathologists remain unfamiliar with the clinical and pathologic features of this disease. The clinical features of NMC sometimes mimic those of other malignancies, and NMC can therefore be overlooked if the diagnosis is not suspected. We present the case of a young male with NMC arising in the mediastinum with elevated serum alpha-fetoprotein levels suggestive of an extragonadal nonseminomatous germ-cell tumor.

Case presentation: A 28-year-old Japanese male presented with cough and left-sided chest pain for 6 weeks. The patient had a mediastinal tumor with metastases to the right lung, lymph nodes, and bones at initial presentation. Nonseminomatous germ cell tumor was suspected due to the young age, location of the tumors, and elevated serum alpha-fetoprotein. However, biopsy confirmed the diagnosis of NMC with immunohistochemistry. The tumor briefly responded to cytotoxic chemotherapy but subsequently progressed and became refractory to the chemotherapy regimen. External beam radiotherapy was administered with dramatic shrinkage of the tumor and a metabolic response on 18-fluoro-2-deoxyglucose positron emission tomography/computed tomography $\left({ }^{18} \mathrm{~F}-\mathrm{FDG}\right.$ PET/CT) scan. However, the patient died 4.5 months after the diagnosis of NMC.

Conclusions: Serum levels of alpha-fetoprotein may be elevated in patients with NMC. Regardless of the level of tumor markers, immunohistochemistry for NUT should be performed in cases of poorly differentiated carcinomas without glandular differentiation arising in the midline structures. ${ }^{18} \mathrm{~F}-\mathrm{FDG}$ PET/CT is useful for staging and assessing responses to therapy.
\end{abstract}

Keywords: NUT midline carcinoma, Tumor markers, Alpha-fetoprotein, Radiotherapy, PET/CT, Case report

\section{Background}

NUT midline carcinoma (NMC) is a highly aggressive subset of squamous cell carcinomas, affecting both children and adults [1]. The genetic hallmark is a rearrangement of the NUT gene, located on chromosome 15 [2]. The rearrangement commonly occurs between the NUT gene and $B E T$ family genes $B R D 4$ and $B R D 3$ [1], although other rare fusion partners of the NUT gene have also been recently reported [3].

Because of the poor prognosis (median survival 6.7 months) [2] and poor response to conventional

\footnotetext{
* Correspondence: yharada@cc.saga-u.ac.jp

1 Department of Medical Oncology, Kameda Medical Center, Kamogawa,

Chiba 296-8602, Japan

${ }^{2}$ Department of Internal Medicine, Division of Haematology, Respiratory Medicine and Oncology, Faculty of Medicine, Saga University, Saga, Japan Full list of author information is available at the end of the article
}

cytotoxic chemotherapy, new drugs such as BET inhibitor (BETi) and histone deacetylase inhibitor ( $\mathrm{HDACi})$ are now in clinical trials for patients with NMC [3]. Because of the availability of these potentially promising new investigational drugs, prompt diagnosis of NMC is even more important to plan appropriate treatment and to encourage patients to consider participating in clinical trials. Most oncologists and pathologists are not familiar with NMC owing to its rarity. The clinical features of NMC sometimes mimic those of other malignancies. For these reasons, NMC may often be misdiagnosed if it is not suspected and specifically looked for. In one study, 114 cases of poorly differentiated carcinomas or unclassified mediastinal malignancies were pathologically reexamined using immunohistochemistry for NUT and fluorescence in situ hybridization (FISH), leading to the diagnosis of NMC in $4(3.5 \%)$ cases [4]. Here we report 
the case of a young male with NMC arising in the mediastinum with elevated serum alpha-fetoprotein (AFP) levels, suggestive of an extra-gonadal nonseminomatous germ cell tumor (NSGCT).

\section{Case presentation}

A 28-year-old Japanese male presented with cough and left-sided chest pain for 6 weeks. The medical, surgical, and family histories were unremarkable. He smoked approximately 20 cigarettes per day for 6 years and infrequently consumed small amounts of alcohol. Physical examination was unremarkable; the lungs were clear to auscultation. Chest X-ray revealed an enlarged mediastinum. A full-body CT scan showed a bulky mediastinal mass with right bronchial stenosis, lymphadenopathy in the right side of the hilum and supraclavicular region, and a mass in the right middle lobe measuring $4.4 \times$ $3.0 \mathrm{~cm}$ (Fig. 1). ${ }^{18} \mathrm{~F}$-FDG PET/CT showed the involvement of multiple bones, including spine, scapula, ribs, sternum, pelvis, and femur (Fig. 2a).

The clinical course and patient background suggested a differential diagnosis that included lung cancer, lymphoma, and a mediastinal germ cell tumor (GCT). Laboratory investigations were significant for an elevated serum lactate dehydrogenase [LDH; 667 IU/L (normal range: 119$229 \mathrm{IU} / \mathrm{L})]$, C-reactive protein $[0.82 \mathrm{mg} / \mathrm{dL}(0.01-0.4 \mathrm{mg} /$ $\mathrm{dL})]$, soluble IL-2 receptor [770 U/mL (112-496 U/mL)], and AFP $[163.8 \mathrm{ng} / \mathrm{mL}(0-20 \mathrm{ng} / \mathrm{mL})]$. Serum levels of $\beta$ human chorionic gonadotropin ( $\beta$-hCG), carcinoembryonic antigen, pro-gastrin-releasing peptide, and cytokeratin-19 fragments (CYFRA) were within normal limits.

Pathology examination of tissue from an endobronchial ultrasound-guided transbronchial needle aspiration (EBUS-TBNA) biopsy of a mediastinal lymph node revealed a loosely cohesive growth pattern with prominent necrosis and degeneration and no clear pattern of differentiation (Fig. 3a). The tumor was composed of ovoid and spindle-shaped cells with anisocytosis, scanty cytoplasm, and irregular ovoid hyperchromatic nuclei (Fig. 3b).

Only a minor proportion $(<5 \%)$ of the cells were positive for AE1/AE3. Squamous cell markers (p40 and p63) were weakly positive in $10-20 \%$ of the cells. Neuroendocrine markers (chromogranin and synaptophysin), leukocyte common antigen, myogenic markers (MyoD1 and myogenin), germ cell markers (placental alkaline phosphatase and hCG), c-kit, TTF-1, CA19-9, and CD30 were all negative. The surface markers of tumor cells obtained with flow cytometry were not compatible with lymphoma. A bone marrow aspiration and biopsy revealed infiltration of cells with pathologic features similar to those of the EBUS-TBNA biopsy specimen.

The clinical presentation of a mediastinal tumor in a young male with an elevated serum AFP suggested NSGCT, but findings of immunohistochemistry of tumor sections were not consistent with that diagnosis. An outside pathology consultation was obtained, and the diagnosis of NMC was suggested due to the pathologic and clinical characteristics. Thus, immunohistochemistry using NUT (C52B1) rabbit monoclonal antibody was performed.

While waiting for results of the immunohistochemical examination for NUT, serum levels of LDH increased to $993 \mathrm{IU} / \mathrm{L}$ on day 11, suggesting rapid disease progression. Because of the poorly differentiated pattern on histologic analysis, elevated serum AFP, and case reports indicating that cisplatin-based treatment showed some efficacy for treating NMC [5], we decided to start chemotherapy with BEP regimen (bleomycin $30 \mathrm{kU}$ on days 1,8 , and 15 ; etoposide, $100 \mathrm{mg} / \mathrm{m}^{2}$ on days $1-5$; and cisplatin, $20 \mathrm{mg} / \mathrm{m}^{2}$ on days $1-5$; given every 21 days). On day 8 of the first cycle, the immunohistochemistry result revealed that most of the neoplastic cell nuclei were strongly positive for NUT in a speckled pattern (Fig. 3c). The diagnosis of NMC was thus confirmed. After two cycles of chemotherapy, CT showed tumor regression, and serum levels of LDH declined. Therefore, the BEP regimen was continued. However, after three cycles, CT showed tumor progression.

Because the performance status of the patient had declined, we considered single-agent chemotherapy to be most appropriate and started doxorubicin $\left(75 \mathrm{mg} / \mathrm{m}^{2}\right.$ every 21 days). Despite the change in chemotherapy regimen, serum levels of LDH continued to increase. Therefore, we judged doxorubicin to be ineffective,

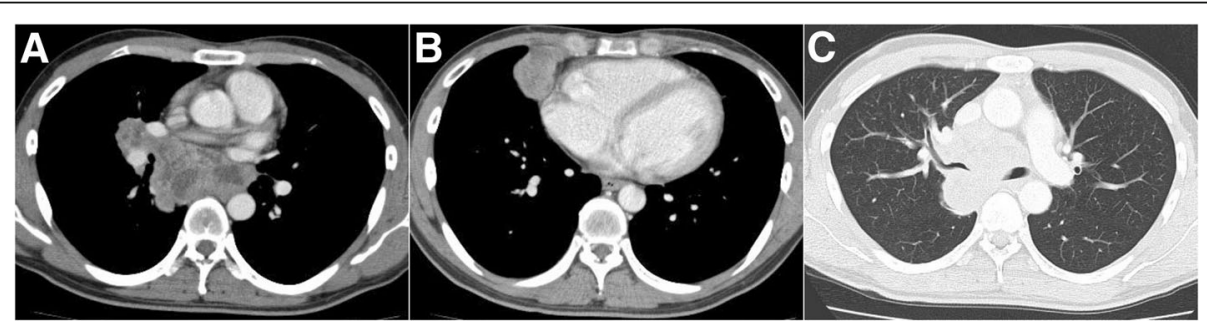

Fig. 1 Full-body CT scan at the first visit. Tumor in the mediastinum and lymphadenopathy in the right side of the hilum and supraclavicular region (a). A tumor in the right middle lobe (b). Right bronchial stenosis due to the mediastinal tumor is shown (c) 


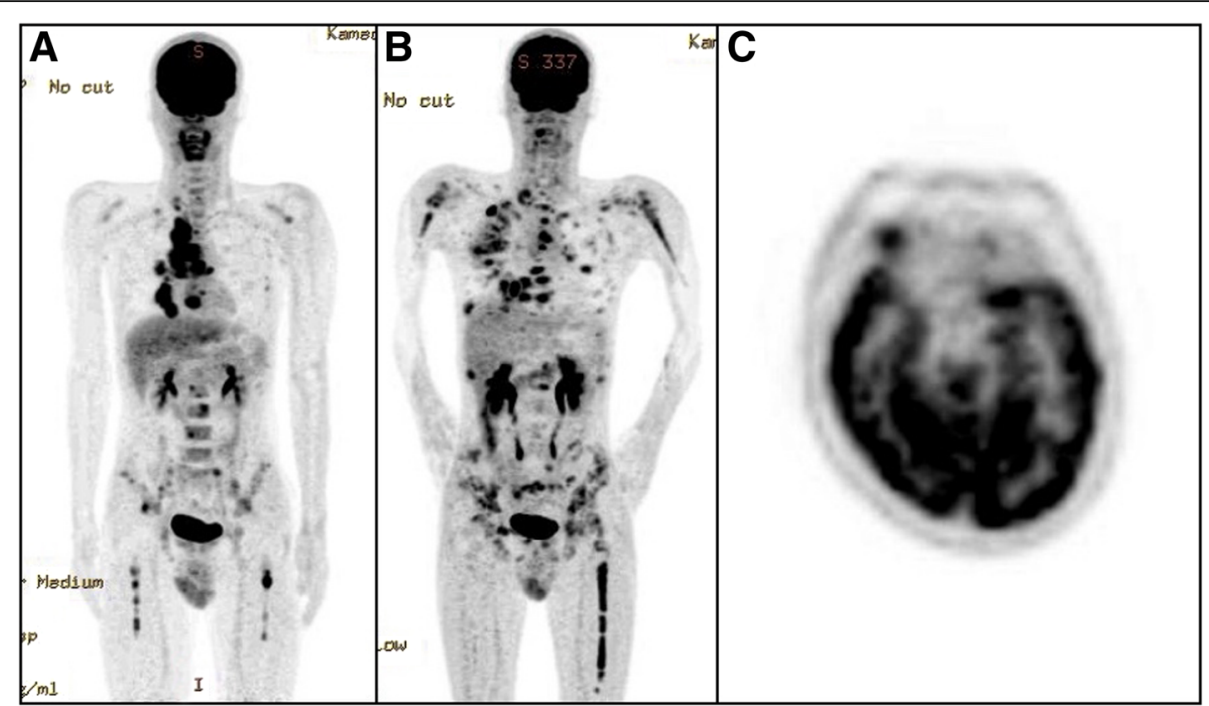

Fig. 2 a ${ }^{18}$ F-FDG PET/CT scan before chemotherapy, with abnormal FDG uptake seen in the mediastinal tumor and the right lung metastasis, lymph nodes, and multiple bones (spine, scapula, ribs, sternum, pelvis, and femur). $\mathbf{b}^{18} \mathrm{~F}$-FDG PET/CT scan after chemotherapy and radiotherapy to the mediastinum, vertebrae L3-S1, and right femur, showing dramatic shrinkage of the tumor and improved metabolic response, while many new abnormal FDG accumulations are demonstrated in other sites. c Abnormal FDG uptake in a mass in the right orbital soft tissues, suggestive of orbital metastasis

although CT scan after the first cycle of doxorubicin showed no change in tumor volume. We believed that local control of the mediastinal mass was most important for the patient at that point to prevent airway obstruction as the tumor progressed. Some authors have reported on the effectiveness of radiotherapy and chemoradiotherapy for NMC $[2,6]$, we therefore administered mediastinal radiotherapy with concomitant weekly docetaxel $\left(30 \mathrm{mg} / \mathrm{m}^{2}\right)$. Radiotherapy was planned with conventional fractionation, $60 \mathrm{~Gy} / 30$ fractions (fr).

CT after 16 Gy had been administered showed an apparent decrease in tumor bulk in the irradiated area, although it had increased in other areas. Docetaxel did not seem to be beneficial for systemic tumor control, and platelet counts had decreased by $2.9 \times 10^{3} / \mu \mathrm{L}$; thus, docetaxel was discontinued after four cycles and radiotherapy alone was continued. The patient started to complain of pain in the lower back and right femur;
MRI confirmed the presence of osteolytic bone metastases. Palliative radiotherapy ( $30 \mathrm{~Gy} / 10 \mathrm{fr}$ ) for metastases in vertebrae L3-S1 and the right femur was concurrently started with irradiation to the mediastinum. Although the pain in the lower back and right femur were relieved, the patient developed painless proptosis in the right eye. While considering additional radiotherapy to prevent pain, we performed ${ }^{18} \mathrm{~F}$-FDG PET/CT to evaluate the extent of metastases to the bones and other organs. It showed that radiotherapy had achieved good local control in the mediastinum, vertebrae L3-S1, and right femur, but there were many new sites of abnormal FDG accumulation (Fig. 2b). Moreover, there was an abnormal FDG uptake in a mass in the right orbital soft tissue (Fig. 2c), suggestive of orbital metastasis. Since then, the patient condition gradually deteriorated, and only palliative care was given. He died 4.5 months after the initial diagnosis of NMC.

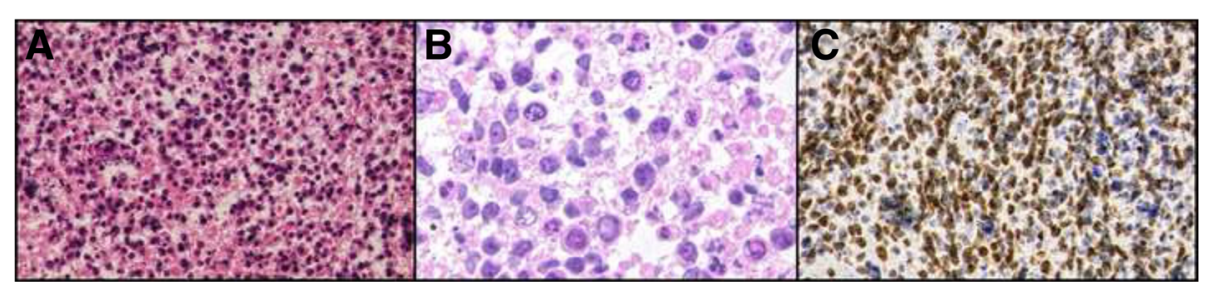

Fig. 3 a Tumor section (H\&E, 100x) with loosely arranged cells with evident necrosis and degeneration and no clear pattern of differentiation. b The tumor is composed of ovoid and spindle-shaped cells with anisocytosis, scanty cytoplasm, and irregular ovoid hyperchromatic nuclei. c Immunohistochemistry of tumor cell nuclei showing speckled staining for NUT 


\section{Discussion}

Except for the inconsistency with histologic results, the characteristics of the present case for the most part resembled those of extra-gonadal NSGCT: 1) occurrence in young adults, mostly males; 2) midline location; 3) metastases to the lungs, liver, and bones; and 4) elevated serum tumor markers (AFP and hCG) [7].

In an international analysis of mediastinal nonseminomas, an elevated serum AFP was present in $74 \%$ (211/ $287)$ and $\beta$-hCG in $38 \%(110 / 287)$ of the cases [8]. The serum AFP level of the present case was compatible with those results. Aside from the present case, there are only three case reports of NMC with elevated serum AFP levels. In one case, the AFP level was $326 \mu \mathrm{g} / \mathrm{L}$ and $\beta$ hCG was <1 IU/L [5]; in another case, they were $62 \mathrm{ng} /$ $\mathrm{mL}$ and N/A [9]; in the other, they were $1742 \mathrm{ng} / \mathrm{mL}$ and $<2 \mathrm{IU} / \mathrm{L}$, respectively [10]. If immunohistochemistry for NUT had not been performed, these cases may have been classified as poorly differentiated carcinoma with midline distribution (extra-gonadal germ cell syndrome), one of the groups of carcinoma of unknown primary with favorable prognosis [11].

French et al. [11] recommended that immunohistochemistry for NUT should be performed in all poorly differentiated carcinomas without glandular differentiation arising in the chest, head, and neck. This means that even if serum tumor markers for GCT are elevated in cases of a tumor arising from such sites, NMC should be suspected.

Definitive diagnosis can be made only by demonstration of nuclear staining using the rabbit monoclonal antibody (clone C52B1) for the NUT protein, even without confirmation of the fusion oncogene with FISH. The antibody is highly sensitive (87\%) and specific (100\%) in non-GCTs [12]. Because we did not suspect NMC initially and did not cryopreserve the pathology specimen, we could not perform the additional molecular analyses such as FISH. It is noteworthy that GCTs can also display nuclear NUT reactivity, but the staining is very focal ( $<5 \%$ of tumor cells) and faint, and it lacks the speckled pattern [13]. In one report, CD34 immunoreactivity was positive in 7 of 11 NMC cases despite they were epithelial tumor [14]. We performed an additional immunohistological staining of CD34 in the present case after the patient's death however, no significant CD34 staining was observed.

Another remarkable feature in the present case was the metastasis to the orbital soft tissues. Only four patients with NMC who had orbital involvement have been reported [2, 15-17]. Although involvement of the mediastinum, paranasal sinuses, nasal cavity, intrathoracic organs, bone, and lymph nodes have been commonly reported, intra-abdominal organs [5, 18] and cutaneous tissue [19] are rare metastatic sites. Till date, there has been no report of brain involvement [20].
The usefulness of ${ }^{18}$ F-FDG PET/CT has been demonstrated in staging and assessing the response to treatment of NMC $[21,22]$. In our patient, ${ }^{18}$ F-FDG PET/CT was performed before and after treatment, showing a good response to radiation therapy by the tumor. Metastasis to the bone marrow was shown with ${ }^{18} \mathrm{~F}-\mathrm{FDG} \mathrm{PET} /$ $\mathrm{CT}$, but not with $\mathrm{CT}$ alone. We therefore recommend performing ${ }^{18} \mathrm{~F}$-FDG $\mathrm{PET} / \mathrm{CT}$ before treatment for accurate staging of NMC.

As in other reports, the tumor in the present case briefly responded to cytotoxic chemotherapy but became refractory to the treatment soon after. There are no specific effective chemotherapeutic regimens [2] because even dose-dense chemotherapy was not found to control the tumor for long [23]. It is probably impossible to cure NMC with cytotoxic chemotherapy alone. Thus, novel targeted therapeutic approaches such as BETi (direct acting inhibitors of the BRD3 and BRD4 bromodomains) and HDACi are highly anticipated [3]. Several phase I clinical trials of BETi (GSK-525762A and OTX015) and HDACi (CUDC-907) are available to patients with NMC. Because of rapid tumor progression and only a short-term response to cytotoxic chemotherapy, we should encourage patients diagnosed with NMC to consider participating in clinical trials as early as possible.

\section{Conclusions}

In summary, we present the case of a patient who had clinical features similar to those of extra-gonadal NSGCT. As serum levels of AFP can be elevated in NMC, immunohistochemistry for NUT should be considered in all poorly differentiated carcinomas arising in midline structures without glandular differentiation, regardless of the levels of tumor markers. ${ }^{18}$ F-FDG PET/ $\mathrm{CT}$ is useful for staging and assessing the response to therapy. It is expected that novel targeted therapies may change the poor prognosis of NMC in the near future.

\section{Abbreviations \\ AFP: Alpha-fetoprotein; BETi: BET inhibitor; EBRT: External beam radiotherapy; EBUS-TBNA: Endobronchial ultrasound-guided transbronchial needle aspiration; FISH: Fluorescence in situ hybridization; fr: fractions; GCT: Germ cell tumor; HDACi: HDAC inhibitor; LDH: Lactate dehydrogenase; NMC: NUT midline carcinoma; NSGCT: Nonseminomatous germ cell tumor; NUT: Nuclear protein in testis; $\beta$-hCG: $\beta$-human chorionic gonadotropin}

\section{Acknowledgements}

We thank the relatives of the patient who kindly gave consent to the publishing of the case.

\section{Funding}

No sources of funding were utilized in the preparation of this report.

Availability of data and materials

All data are presented in the manuscript.

Authors' contributions

$\mathrm{YH}$, the corresponding author was the treating physician and prepared the manuscript. KT was the consultant histopathologist and performed the 
immunohistochemistry for NUT. KS was involved in the patient's radiation therapy. $\mathrm{KH}$ is the histopathologist and contributed to the histopathological details of the manuscript. TK and YO were involved in the patient's management and revised the article for important intellectual content. All authors read and approved the final manuscript.

\section{Competing interests}

The authors declare that they have no competing interests.

\section{Consent for publication}

Written informed consent for publication of their clinical details and clinical images was obtained from the relatives of the patient. A copy of the written consent is available for review by the editor of the journal.

\section{Ethics approval and consent to participate}

Not applicable.

\section{Author details}

'Department of Medical Oncology, Kameda Medical Center, Kamogawa, Chiba 296-8602, Japan. ${ }^{2}$ Department of Internal Medicine, Division of Haematology, Respiratory Medicine and Oncology, Faculty of Medicine, Saga University, Saga, Japan. ${ }^{3}$ Pathology Project for Molecular Targets, the Cancer Institute, Japanese Foundation for Cancer Research, Tokyo, Japan. ${ }^{4}$ Division of Pathology, the Cancer Institute, Japanese Foundation for Cancer Research, Tokyo, Japan. ${ }^{5}$ Department of Radiation Therapy, Kameda Medical Center, Kamogawa, Chiba 296-8602, Japan. ${ }^{6}$ Department of Diagnostic Pathology, Kameda Medical Center, Kamogawa, Chiba 296-8602, Japan.

Received: 9 March 2016 Accepted: 9 November 2016 Published online: 17 November 2016

\section{References}

1. French CA, Ramirez CL, Kolmakova J, Hickman TT, Cameron MJ, Thyne ME, et al. BRD-NUT oncoproteins: a family of closely related nuclear proteins that block epithelial differentiation and maintain the growth of carcinoma cells. Oncogene. 2008;27(15):2237-42.

2. Bauer DE, Mitchell CM, Strait KM, Lathan CS, Stelow EB, Luer SC, et al. Clinicopathologic features and long-term outcomes of NUT midline carcinoma. Clin Cancer Res. 2012;18(20):5773-9.

3. French CA, Rahman S, Walsh EM, Kuhnle S, Grayson AR, Lemieux ME, et al. NSD3-NUT fusion oncoprotein in NUT midline carcinoma: implications for a novel oncogenic mechanism. Cancer Discov. 2014;4(8):928-41.

4. Evans AG, French CA, Cameron MJ, Fletcher CD, Jackman DM, Lathan CS, et al. Pathologic characteristics of NUT midline carcinoma arising in the mediastinum. Am J Surg Pathol. 2012;36(8):1222-7.

5. Ball A, Bromley A, Glaze S, French CA, Ghatage P, Kobel M. A rare case of NUT midline carcinoma. Gynecol Oncol Case Rep. 2012;3:1-3.

6. Engleson J, Soller M, Panagopoulos I, Dahlen A, Dictor M, Jerkeman M. Midline carcinoma with t(15;19) and BRD4-NUT fusion oncogene in a 30year-old female with response to docetaxel and radiotherapy. BMC Cancer. 2006;6:69.

7. Mizushima Y. Extragonadal germ cell tumors. Internal Med (Tokyo, Japan). 2004:43(12):1099-100.

8. Bokemeyer C, Nichols CR, Droz JP, Schmoll HJ, Horwich A, Gerl A, et al. Extragonadal germ cell tumors of the mediastinum and retroperitoneum: results from an international analysis. J Clin Oncol. 2002;20(7):1864-73.

9. Parikh SA, French CA, Costello BA, Marks RS, Dronca RS, Nerby CL, et al. NUT midline carcinoma: an aggressive intrathoracic neoplasm. J Thorac Oncol. 2013;8(10):1335-8.

10. Raza A, Cao H, Conrad R, Cobb C, Castelino-Prabhu S, Mirshahidi S, et al. Nuclear protein in testis midline carcinoma with unusual elevation of alphafetoprotein and synaptophysin positivity: a case report and review of the literature. Expert Rev Anticancer Ther. 2015;15(10):1199-213.

11. Pavlidis N, Fizazi K. Carcinoma of unknown primary (CUP). Crit Rev Oncol Hematol. 2009;69(3):271-8.

12. Haack H, Johnson LA, Fry CJ, Crosby K, Polakiewicz RD, Stelow EB, et al. Diagnosis of NUT midline carcinoma using a NUT-specific monoclonal antibody. Am J Surg Pathol. 2009;33(7):984-91.

13. French CA. The importance of diagnosing NUT midline carcinoma. Head Neck Pathol. 2013;7(1):11-6.
14. French CA, Kutok JL, Faquin WC, Toretsky JA, Antonescu CR, Griffin CA, et al. Midline carcinoma of children and young adults with NUT rearrangement. J Clin Oncol. 2004;22(20):4135-9.

15. D'Souza JN, Notz G, Bogdasarian RN, Cognetti DM, Curry JM, Rosen MR, et al. Orbital Involvement by NUT Midline Carcinoma. Ophthal Plast Reconstr Surg. 2015;31(6):e147-50.

16. Stirnweiss A, McCarthy K, Oommen J, Crook ML, Hardy K, Kees UR, et al. A novel BRD4-NUT fusion in an undifferentiated sinonasal tumor highlights alternative splicing as a contributing oncogenic factor in NUT midline carcinoma. Oncogenesis. 2015;4:e174.

17. Stelow EB. A review of NUT midline carcinoma. Head Neck Pathol. 2011;5(1):31-5.

18. Shehata BM, Steelman CK, Abramowsky CR, Olson TA, French CA, Saxe DF, et al. NUT midline carcinoma in a newborn with multiorgan disseminated tumor and a 2-year-old with a pancreatic/hepatic primary. Pediatr Dev Pathol. 2010;13(6):481-5.

19. Young MR, Millington $K$, Clarke LE, Helm K. NUT midline carcinoma with cutaneous metastases. J Am Acad Dermatol. 2012;67(2):323-4.

20. Sholl LM, Nishino M, Pokharel S, Mino-Kenudson M, French CA, Janne PA, et al. Primary pulmonary NUT midline carcinoma: clinical, radiographic, and pathologic characterizations. J Thorac Oncol. 2015;10(6):951-9.

21. Ciftci E, Demirsoy U, Anik Y, Gorur G, Corapcioglu F, Demir H. Staging and evaluation of neoadjuvant chemotherapy response with (1)(8)F-FDG PET/CT in NUT-midline carcinoma in a child: a case report and review of the literature. Revista espanola de medicina nuclear e imagen molecular. 2015;34(1):53-5.

22. Shaikh F, Pagedar N, Awan O, McNeely P. Sinonasal NUT-midline carcinoma - a multimodality approach to diagnosis, staging and post-surgical restaging. Cureus. 2015;7(7):e288.

23. Maur M, Toss A, Dominici M, Frassoldati A, Corradini P, Maiorana A, Fontana $A$, et al. Impressive response to dose-dense chemotherapy in a patient with NUT midline carcinoma. Am J Case Rep. 2015;16:424-9.

\section{Submit your next manuscript to BioMed Central and we will help you at every step:}

- We accept pre-submission inquiries

- Our selector tool helps you to find the most relevant journal

- We provide round the clock customer support

- Convenient online submission

- Thorough peer review

- Inclusion in PubMed and all major indexing services

- Maximum visibility for your research

Submit your manuscript at www.biomedcentral.com/submit
Biomed Central 